\title{
Analysis of preconditioning and multigrid for Euler flows with low-subsonic regions*
}

\author{
B. Koren \\ Centrum voor Wiskunde en Informatica, Afdeling Numerieke Wiskunde, P.O. Box 94079 , \\ 1090 GB Amsterdam. The Netherlands
}

B. van Leer

The University of Michigan, Department of Aerospace Engineering, 1320 Beal Avenue, Ann Arbor, MI 48109-2118, USA

\begin{abstract}
For subsonic flows and upwind-discretized, linearized 1-D Euler equations, the smoothing behavior of multigrid-accelerated point Gauss-Seidel relaxation is analyzed. Error decay by convection across domain boundaries is also discussed. A fix to poor convergence rates at low Mach numbers is sought in replacing the point relaxation applied to unconditioned Euler equations, by locally implicit "time"-stepping applied to preconditioned Euler equations. The locally implicit iteration step is optimized for good damping of high-frequency errors. Numerical inaccuracy at low Mach numbers is also addressed.
\end{abstract}

Keywords: Subsonic flows, Euler equations, multigrid methods, preconditioning matrices, convergence and accuracy analyses.

AMS subject classification: $65 \mathrm{~N} 12,65 \mathrm{~N} 15,65 \mathrm{~N} 22,65 \mathrm{~N} 55,76 \mathrm{G} 25,76 \mathrm{M} 25$.

\section{Introduction}

The mathematical theory of inviscid, subsonic gas flows is relatively undeveloped in comparison with that of inviscid, transonic, supersonic and hypersonic flows. An indication of this is the small amount of literature which is available on the mathematical aspects of subsonic gas dynamics. Whereas various thorough text books exist, which extensively deal with the mathematics of transonic, supersonic or hypersonic gas dynamics (see e.g. $[3,5,8,17,20]$ ), for the subsonic case we only know a few book chapters (chapters 2 and 3 from [1], and chapter 2 from [16]). At present, mathematical research in the inviscid, subsonic flow regime is at a rapid pace, particularly as far as it concerns numerical computations in the zero Mach-number limit. The present paper is intended to contribute to this

* The work reported was performed in the framework of the BRITE-EURAM Aeronautics R\&D Programme of the European Communities (Contract No. AER2-CT92-0040). The work was started during the second author's visit to CWI in 1993.

(C) J.C. Baltzer AG, Science Publishers 
development. In it, the flows of interest are not flows with uniformly low Mach numbers (i.e. flows with $M \ll 1$ throughout almost the entire computational domain), but flows with locally low Mach numbers (flows with small stagnation regions and - particularly - for Navier-Stokes extensions: flows with thin boundary layers and wakes). We also remark that in our case the pressure cannot become infinitely large for $M \downarrow 0$. (The pressure can become infinitely large for $M \downarrow 0$ when the velocity remains finite but the speed of sound becomes infinite $[9,10]$.) We consider the case in which the pressure remains finite for $M \downarrow 0$, the case in which - as opposed to in the preceding incompressible one - the velocity goes to zero but the speed of sound remains finite (which is physically more realistic in e.g. aircraftaerodynamics applications).

Since about a decade, various multigrid methods exist that give good convergence rates for steady Euler-flow computations at high-subsonic inflow Mach numbers (see chapter 9 from [26] for an overview). For decreasing inflow Mach numbers, or enlarging low-subsonic flow regions, convergence rates are known to deteriorate. This decrease is not specific for multigrid methods, but seems to hold for any solution method. The cause has to be sought in the continuous Euler equations, in their increasing stiffness (i.e. in their increasing disparity of wave speeds) at decreasing subsonic Mach numbers. With the application of single-grid, explicit time-stepping schemes in mind, various fixes have already been proposed to this stiffness problem. See [23] for a review of this. A key paper is [14]. In it, a preconditioning matrix is given which completely equalizes the three wave speeds $(u-c, u$ and $u+c)$ of the 1-D Euler equations. Furthermore, the paper gives preconditioning matrices for the 2-D and 3-D Euler equations.

Besides convergence problems, for decreasing Mach numbers also accuracy problems arise $[24,25]$. Whereas the convergence problems are intrinsically related to the continuous Euler equations (to their stiffness), the accuracy problems hold for the discretized equations (independent of whether the discretization is central or upwind).

In the present paper, we will mainly focus on the stiffness problem. It is expected that solution methods other than explicit time-stepping schemes may also profit from preconditioning matrices such as those proposed in [14]; some early experiences with e.g. Approximate Factorization were reported by Godfrey et al. [6]. In this paper we will optimize a multigrid accelerated, locally implicit iteration method, applied to subsonic, preconditioned Euler equations. To start with, in section 2, the continuous unconditioned equations and their discretization are introduced. In section 3, first a smoothing analysis is given of point Gauss-Seidel relaxation applied to these unconditioned discrete equations, and next a discussion is made of error convection across domain boundaries. It is shown that for low Mach numbers the convergence properties are poor. In section 4 it is made clear that for flows with uniformly low Mach numbers, numerical accuracy may be poor as well. In section 5 a $1-D$ preconditioning matrix is derived which is particularly capable of removing stiffness. In section 6 we discuss a simple way to 
implement the preconditioning, without collecting the accuracy benefit. At the end of section 6 we arrive at the discrete, preconditioned system to be solved. The system contains a free parameter: a locally implicit iteration step-size, which is optimized for smoothing. The optimization is done in section 7 , through local-mode analysis applied to the upwind-discretized, linearized, preconditioned 1-D Euler equations. In section 8 the error smoothing and error convection of the locally implicit iteration are analyzed.

\section{The equations}

\subsection{Continuous equations}

Consider the 1-D Euler equations

$$
\frac{\partial Q}{\partial t}+\frac{\partial f(Q)}{\partial x}=0
$$

with $Q$ the conservative state vector

$$
Q=\left(\begin{array}{c}
\rho \\
\rho u \\
\rho e
\end{array}\right)
$$

$f(Q)$ the corresponding flux vector

$$
f(Q)=\left(\begin{array}{c}
\rho u \\
\rho u^{2}+p \\
\rho u(e+p / \rho)
\end{array}\right)
$$

and $e$ the internal energy, which for a perfect gas reads

$$
e=\frac{1}{\gamma-1} \frac{p}{\rho}+\frac{1}{2} u^{2}
$$

Linearization of (1a) with respect to the conservative variables yields

$$
\frac{\mathrm{d} f}{\mathrm{~d} Q}=\left(\begin{array}{ccc}
\frac{\partial Q}{\partial t}+\frac{\mathrm{d} f}{\mathrm{~d} Q} \frac{\partial Q}{\partial x}=0 \\
0 & 1 & 0 \\
\frac{\gamma-3}{2} u^{2} & (3-\gamma) u & \gamma-1 \\
\frac{\gamma-2}{2} u^{3}-\frac{1}{\gamma-1} u c^{2} & \frac{(3-2 \gamma)}{2} u^{2}+\frac{1}{\gamma-1} c^{2} & \gamma u
\end{array}\right),
$$


where $c^{2}=\sqrt{\gamma p / \rho}$. In order to simplify the analysis, following Turkel [22], the transformation from conservative variables $Q$ to non-conservative (entropy) variables $q$ is made:

$$
\mathrm{d} q \equiv\left(\begin{array}{c}
\frac{1}{\rho c} \mathrm{~d} p \\
\mathrm{~d} u \\
\mathrm{~d} p-c^{2} \mathrm{~d} \rho
\end{array}\right) .
$$

(For viscous flows - instead of these entropy variables - Choi and Merkle [2] recommend the use of temperature variables: $(p, u, T)^{\mathrm{T}}$.) The transformation matrix corresponding to the entropy variables,

$$
\frac{\mathrm{d} Q}{\mathrm{~d} q}=\left(\begin{array}{ccc}
\frac{\rho}{c} & 0 & -\frac{1}{c^{2}} \\
\frac{\rho u}{c} & \rho & -\frac{u}{c^{2}} \\
\frac{1}{2} \frac{\rho u^{2}}{c}+\frac{1}{\gamma-1} \rho c & \rho u & -\frac{1}{2} \frac{u^{2}}{c^{2}}
\end{array}\right),
$$

brings equation ( $1 \mathrm{a})$ into the analytically more tractable form

$$
\begin{gathered}
\frac{\partial q}{\partial t}+A \frac{\partial q}{\partial x}=0 \\
A=\frac{\mathrm{d} q}{\mathrm{~d} Q} \frac{\mathrm{d} f}{\mathrm{~d} Q} \frac{\mathrm{d} Q}{\mathrm{~d} q}=\left(\begin{array}{lll}
u & c & 0 \\
c & u & 0 \\
0 & 0 & u
\end{array}\right) .
\end{gathered}
$$

\subsection{Discrete equations}

For simplicity, we assume $A$ to be constant and next make a first-order upwind, cell-centered finite-volume discretization of the space operator in (5a). Then the semi-discrete equation in cell $\Omega_{i}$ (with mesh size $h$ ) reads

$$
h \frac{\partial q_{i}}{\partial t}+A^{+}\left(q_{i}-q_{i-1}\right)+A^{-}\left(q_{i+1}-q_{i}\right)=0,
$$

with $i$ running in the positive $x$-direction, and with $A^{+}$and $A^{-}$the matrices corresponding to the positive and negative eigenvalues of matrix $A$ :

$$
\begin{aligned}
& A^{+}=R_{A} \Lambda_{A}^{+} R_{A}^{-1}, \\
& A^{-}=R_{A} \Lambda_{A}^{-} R_{A}^{-1} .
\end{aligned}
$$

With $\Lambda_{A}=\operatorname{diag}(u-c, u, u+c)$, we get

$$
R_{A}=\left(\begin{array}{rrr}
1 & 0 & 1 \\
-1 & 0 & 1 \\
0 & 1 & 0
\end{array}\right)
$$


and hence for subsonic flow in the positive $x$-direction $(0<u<c$, without loss of generality we assume this to hold throughout the paper):

$$
\begin{aligned}
& A^{+}=\frac{1}{2}\left(\begin{array}{ccc}
u+c & u+c & 0 \\
u+c & u+c & 0 \\
0 & 0 & 2 u
\end{array}\right), \\
& A^{-}=\frac{1}{2}\left(\begin{array}{ccc}
u-c & c-u & 0 \\
c-u & u-c & 0 \\
0 & 0 & 0
\end{array}\right) .
\end{aligned}
$$

\section{Convergence}

\subsection{Convergence through error smoothing}

When one applies point Gauss-Seidel relaxation to find the steady solution of (6), for successively downstream and upstream relaxation sweeps, the iteration formulae are

$$
\begin{aligned}
|A|\left(q_{i}^{n+1}-q_{i}^{n}\right) & =-A^{+}\left(q_{i}^{n}-q_{i-1}^{n+1}\right)-A^{-}\left(q_{i+1}^{n}-q_{i}^{n}\right), \\
|A|\left(q_{i}^{n+2}-q_{i}^{n+1}\right) & =-A^{+}\left(q_{i}^{n+1}-q_{i-1}^{n+1}\right)-A^{-}\left(q_{i+1}^{n+2}-q_{i}^{n+1}\right),
\end{aligned}
$$

with $|A| \equiv A^{+}-A^{-}$and $n$ the relaxation sweep counter. In order to investigate the smoothing properties we introduce the local solution error

$$
\delta_{i}^{n}=q_{i}^{*}-q_{i}^{n},
$$

and the Fourier form

$$
\delta_{i}^{n}=D^{n} \mathrm{e}^{\mathrm{i} \theta i}, \quad|\theta| \in\left[\frac{\pi}{2}, \pi\right],
$$

with $q_{i}^{*}$ the exact local solution, $D^{n}$ the amplitude vector $\left(D_{1}^{n}, D_{2}^{n}, D_{3}^{n}\right)$ and $\mathrm{e}^{\mathrm{i} \theta i}$ the (scalar) mode. With (1la) and (11b), it follows for the amplification matrices $\mathscr{M}_{\text {downstream }}$ and $\mathscr{M}_{\text {upstream }}$ corresponding with (10a) and (10b), respectively:

$$
\begin{aligned}
\mathscr{M}_{\text {downstream }} & =-\left(-\mathrm{e}^{-\mathrm{i} \theta} A^{+}+|A|\right)^{-1} \mathrm{e}^{\mathrm{i} \theta} A^{-}, \\
\mathscr{M}_{\text {upstream }} & =\left(\mathrm{e}^{\mathrm{i} \theta} A^{-}+|A|\right)^{-1} \mathrm{e}^{-\mathrm{i} \theta} A^{+} .
\end{aligned}
$$

By substitution of $A^{+}$and $A^{-}$we find the solution-independent matrices

$$
\begin{aligned}
\mathscr{M}_{\text {downstream }} & =\frac{1}{2}\left(\begin{array}{ccc}
\mathrm{e}^{\mathrm{i} \theta} & -\mathrm{e}^{\mathrm{i} \theta} & 0 \\
-\mathrm{e}^{\mathrm{i} \theta} & \mathrm{e}^{\mathrm{i} \theta} & 0 \\
0 & 0 & 0
\end{array}\right), \\
\mathscr{M}_{\text {upstream }} & =\frac{1}{2}\left(\begin{array}{ccc}
\mathrm{e}^{-\mathrm{i} \theta} & \mathrm{e}^{-\mathrm{i} \theta} & 0 \\
\mathrm{e}^{-\mathrm{i} \theta} & \mathrm{e}^{-\mathrm{i} \theta} & 0 \\
0 & 0 & 2 \mathrm{e}^{-\mathrm{i} \theta}
\end{array}\right),
\end{aligned}
$$


with spectral radii

$$
\begin{aligned}
\rho\left(\mathscr{M}_{\text {downstream }}\right) & =\left|\mathrm{e}^{\mathrm{i} \theta}\right|=1, \quad \forall|\theta| \in\left[\frac{\pi}{2}, \pi\right], \\
\rho\left(\mathscr{M}_{\text {upstream }}\right) & =\left|\mathrm{e}^{-\mathrm{i} \theta}\right|=1, \quad \forall|\theta| \in\left[\frac{\pi}{2}, \pi\right] .
\end{aligned}
$$

(Since the matrices (13a) and (13b) are symmetric, the spectral norms, which determine the smoothing properties for $n=1$, are identical to the spectral radii.) Note that in the case of a symmetric sweep, according to this Fourier analysis, one has perfect smoothing: $\mathscr{M}_{\text {upstream }} \mathscr{M}_{\text {downstream }}=0$. However, in the case of subsonic flow with non-periodic boundary conditions, one generally has error reflections at the outflow boundary when still iterating. Therefore this theoretical, perfect smoothing result is not realistic and therefore we prefer to consider the downstream and upstream amplification matrices separately. However, for the two separate sweeps, the smoothing factors (14a) and (14b) are surprising as well. They seem to be in contradiction with numerical findings; for e.g. standard, high-subsonic airfoil-flow computations, one generally observes good multigrid convergence. A first explanation of this apparent contradictory result is that care has to be taken in interpreting (14a) and (14b); the frozen coefficients assumption generally loses its validity for high-subsonic Mach numbers. As opposed to this, for low-subsonic Mach numbers it seems a reasonable assumption (e.g., for $\lim _{M \downarrow 0}, \rho$ becomes constant).

\subsection{Convergence through error convection across domain boundaries}

A second explanation of the seeming contradictory convergence estimate for high-subsonic flows in the general case of non-periodic boundary conditions is that for the downstream and upstream sweeps separately, local-mode analysis solely is just too pessimistic. For non-periodic, high-subsonic flow computations, additional error decay through advection over the domain boundaries may be significant and may therefore not be neglected. Note herewith that point Gauss-Seidel relaxation can be interpreted as locally implicit timestepping at an infinitely large time step, which with non-zero wave propagation speeds $u-c, u$ and $u+c$, implies a significant beneficial influence on convergence. This phenomenon of solution errors being expelled out of the computational domain by convection may next further explain the poor multigrid performance for low-subsonic flows. In spite of the infinitely large time step associated with point Gauss-Seidel relaxation, for $\lim _{M 10}$, the propagation of entropy errors, and therefore their expulsion, may well start to stagnate. See [11] for a convergence analysis in which error convection across domain boundaries is considered. 


\section{Accuracy}

\subsection{Well-posedness of the continuous equations}

For $\lim _{M \downarrow 0}$, exact solutions of the continuous Euler equations are assumed to converge to the corresponding, exact, incompressible flow solutions. (Compressible flow in the zero Mach-number limit is assumed to be a regular perturbation of incompressible flow.) As a support for this, see e.g. the perturbation theory analysis of slightly compressible flow past a circle in chapter 2 of [4]. The limit $\lim _{M \downarrow 0}$ is not known to cause general non-uniqueness problems; as opposed to for $\lim _{M \rightarrow 1}$ (see Morawetz [18]), for $\lim _{M \downarrow 0}$ boundary-value problems are not known to become ill-posed.

\subsection{Inaccuracy of the discrete equations}

In the discrete case accuracy problems arise for $\lim _{M \downarrow 0}$. The inaccuracy can be analyzed through the conservative 1-D Euler equations discretized through e.g. a first-order accurate flux-difference splitting scheme (such as Osher's [19] or Roe's [21]). In non-conservative form the corresponding modified equation reads

$$
\frac{\partial q}{\partial t}+A \frac{\partial q}{\partial x}=\frac{h}{2}\left[\frac{\partial}{\partial x}\left(|A| \frac{\partial q}{\partial x}\right)-\frac{\partial}{\partial x}\left(\frac{\mathrm{d} q}{\mathrm{~d} Q}\right) \frac{\mathrm{d} Q}{\mathrm{~d} q}|A| \frac{\partial q}{\partial x}\right]
$$

With

$$
|A|=\left(\begin{array}{lll}
c & u & 0 \\
u & c & 0 \\
0 & 0 & u
\end{array}\right),
$$

with $\mathrm{d} Q / \mathrm{d} q$ according to (4), and with $\mathrm{d} q / \mathrm{d} Q=(\mathrm{d} Q / \mathrm{d} q)^{-1}$, the two numerical diffusion terms in the right-hand side of (15) can be written out as

$$
\begin{aligned}
\frac{\partial}{\partial x}\left(|A| \frac{\partial q}{\partial x}\right)= & \frac{\partial u}{\partial x}\left(\begin{array}{ccc}
\frac{1}{M} & 1 & 0 \\
1 & \frac{1}{M} & 0 \\
0 & 0 & 1
\end{array}\right) \frac{\partial q}{\partial x}+u \frac{\partial}{\partial x}\left(\begin{array}{ccc}
\frac{1}{M} & 1 & 0 \\
1 & \frac{1}{M} & 0 \\
0 & 0 & 1
\end{array}\right) \frac{\partial q}{\partial x} \\
& +c\left(\begin{array}{ccc}
1 & M & 0 \\
M & 1 & 0 \\
0 & 0 & M
\end{array}\right) \frac{\partial^{2} q}{\partial x^{2}}
\end{aligned}
$$




$$
-\left(\begin{array}{ccc}
\frac{\partial}{\partial x}\left(\frac{\mathrm{d} q}{\mathrm{~d} Q}\right) \frac{\mathrm{d} Q}{\mathrm{~d} q}|A| \frac{\partial q}{\partial x}= & \\
\frac{c}{\rho} \frac{\partial \rho}{\partial x}+(\gamma-1) M \frac{\partial u}{\partial x}+\frac{\partial c}{\partial x} & \frac{u}{\rho} \frac{\partial \rho}{\partial x}+(\gamma-1) \frac{\partial u}{\partial x}+M \frac{\partial c}{\partial x} & 0 \\
\frac{u}{\rho} \frac{\partial \rho}{\partial x}+\frac{\partial u}{\partial x} & \frac{c}{\rho} \frac{\partial \rho}{\partial x}+M \frac{\partial u}{\partial x} & -\frac{M}{\rho c} \frac{\partial u}{\partial x} \\
\frac{1}{\gamma-1} \rho u \frac{\partial u}{\partial x}+2 \rho c \frac{\partial c}{\partial x} & \frac{1}{\gamma-1} \rho c \frac{\partial u}{\partial x}+2 \rho u \frac{\partial c}{\partial x} & -2 M \frac{\partial c}{\partial x}
\end{array}\right) \frac{\partial q}{\partial x}
$$

It appears that for $\lim _{M \downarrow 0}$, with $h$ fixed and with all three components of $\partial q / \partial x$ finite, the first two vector elements of

$$
\frac{\partial u}{\partial x}\left(\begin{array}{ccc}
\frac{1}{M} & 1 & 0 \\
1 & \frac{1}{M} & 0 \\
0 & 0 & 1
\end{array}\right) \frac{\partial q}{\partial x}
$$

become infinitely large. (All other diffusion terms become finite or zero.)

\section{Preconditioning}

\subsection{Removing the stiffness}

For a detailed account of this topic we refer to [14]. The ratio of the maximum and minimum characteristic wave speeds (the characteristic condition number $K$ ) of $A$ can be written as

$$
K_{A}(M)=\max \left(\frac{1+M}{M}, \frac{1+M}{1-M}\right), \quad M \equiv \frac{u}{c} \in(0,1),
$$

see also figure 1. At $M=0$ and $M=1, A$ is singular. Preconditioning $A$ (by premultiplying it) with the $3 \times 3$-matrix $P$ transforms equation (5a) into

$$
\frac{\partial q}{\partial t}+P A \frac{\partial q}{\partial x}=0
$$

For general $P$, the possibility of doing time-accurate calculations is lost. When solving steady problems, this is of no concern. $P$ should at least be invertible and should remove the static and sonic singularity. In the ideal case, $P$ leads to the situation: (i) that $K_{P A}(M)=1$ over the entire subsonic Mach-number range, and (ii) that $P A$ yields two downstream waves and one upstream wave. Satisfaction of the second property, conservation of the propagation directions of the three waves, avoids the complication of a change of numbers of boundary conditions 


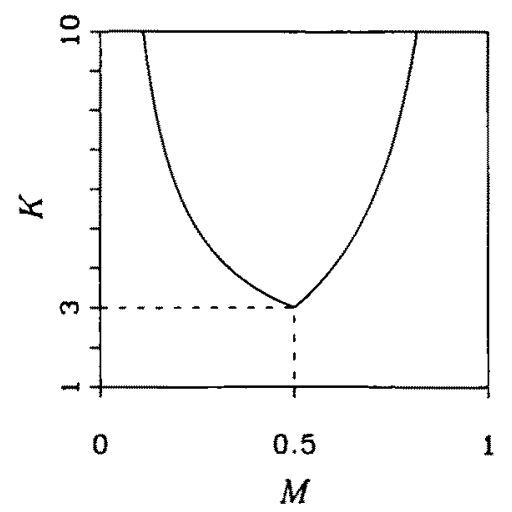

Figure 1. Characteristic condition of the derivative matrix $A$ as a function of the Mach number.

to be imposed at in- and outlet. This property is satisfied by taking $P$ positive definite, which implies that $P$ must be symmetric.

A common choice for $P$ is

$$
P=w|A|^{-1}
$$

with $w$ some arbitrary propagation speed that can still be chosen. With (20) one has $\Lambda_{P A}=\operatorname{diag}(-w, w, w)$. In multi-D, perfect subsonic preconditioning is not possible. For 2-D subsonic Euler flows and for $\mathrm{d} q=\left((1 / \rho c) \mathrm{d} p, \mathrm{~d} u, \mathrm{~d} v, \mathrm{~d} p-c^{2} \mathrm{~d} \rho\right)^{T}$, the following preconditioning matrix is proposed in [14]:

$$
P=\left(\begin{array}{cccc}
\frac{M^{2}}{\sqrt{1-M^{2}}} & \frac{-M}{\sqrt{1-M^{2}}} & 0 & 0 \\
\frac{-M}{\sqrt{1-M^{2}}} & \frac{1}{\sqrt{1-M^{2}}}+1 & 0 & 0 \\
0 & 0 & \sqrt{1-M^{2}} & 0 \\
0 & 0 & 0 & 1
\end{array}\right) .
$$

For 2-D stagnation flows, preconditioning by (20) may lead to stability problems; inside stagnation regions instability may occur due to the great flow-angle sensitivity. In [15] a fix is proposed to these problems. The fix consists of preconditioning through the sub-optimal, but for stagnation flows stable matrix

$$
P=\left(\begin{array}{cccc}
\alpha \frac{M^{2}}{\sqrt{1-M^{2}}} & -\alpha \frac{M}{\sqrt{1-M^{2}}} & 0 & 0 \\
-\alpha \frac{M}{\sqrt{1-M^{2}}} & \alpha\left(\frac{1}{\sqrt{1-M^{2}}}+1\right) & 0 & 0 \\
0 & 0 & \sqrt{1-M^{2}} & 0 \\
0 & 0 & 0 & \alpha
\end{array}\right),
$$




$$
\begin{aligned}
\alpha=\frac{1}{2}, & 0 \leq M \leq \frac{1}{3}, \\
\alpha=\frac{3}{4}\left[1+3\left(M-\frac{1}{2}\right)\left(1-12\left(M-\frac{1}{2}\right)^{2}\right)\right], & \frac{1}{3}<M<\frac{2}{3}, \\
\alpha=1, & \frac{2}{3} \leq M<1 .
\end{aligned}
$$

Note the good resemblance between (21) and (22). Since local-mode analysis for the preconditioned full, 2-D Euler equations is hard and does not lead to transparent results, we do the analysis for the 1-D Euler equations, with as preconditioning matrix a single 1-D version of both 2-D $P$ 's (21) and (22). This 1-D preconditioning matrix is already given in section 6.3 of [13]. We proceed by re-deriving it.

Striving for the almost diagonal form

$$
P A=\left(\begin{array}{rrr}
-w & 0 & 0 \\
\hat{w} & w & 0 \\
0 & 0 & w
\end{array}\right),
$$

which still satisfies $K_{P A}(M)=1, \forall M \in(0,1)$, a symmetric 1-D version of (21) and (22) can be found. For $w=u$, it follows

$$
P=\left(\begin{array}{ccc}
\frac{M^{2}}{1-M^{2}} & \frac{-M}{1-M^{2}} & 0 \\
\frac{-M}{1-M^{2}} & \frac{1}{1-M^{2}}+1 & 0 \\
0 & 0 & 1
\end{array}\right)
$$

(and, unimportant, $\hat{w}=2 c$ ). Note that $P$ according to (24) is positive-definite; for $M \in(0,1)$ its three eigenvalues are all positive:

$$
\lambda_{1}=1 \quad \text { and } \quad \lambda_{2,3}=\frac{1 \pm \sqrt{1-M^{2}+M^{4}}}{1-M^{2}}>0, \quad \forall M \in(0,1) .
$$

Also still note the freedom in the derivation of this preconditioning matrix. E.g., another $w$ could have been chosen; $w=u+c$ would have yielded

$$
P=\left(\begin{array}{ccc}
\frac{M}{1-M} & \frac{-1}{1-M} & 0 \\
\frac{-1}{1-M} & \frac{2-M^{2}}{M(1-M)} & 0 \\
0 & 0 & \frac{1+M}{M}
\end{array}\right)
$$

Moreover, instead of preconditioning, postconditioning could have been applied. The difference between pre- and postconditioning can be clarified by considering the auxiliary equation $A \mathrm{~d} q / \mathrm{d} x=r$. Preconditioning this equation $(P A \mathrm{~d} q / \mathrm{d} x=r)$ is identical to right-hand side transformation $\left(A \mathrm{~d} q / \mathrm{d} x=P^{-1} r\right)$, 
whereas postconditioning $(A P \mathrm{~d} q / \mathrm{d} x=r)$ can be interpreted as solution transformation. Postconditioning (5a) by a symmetric $P$ such that

$$
A P=\left(\begin{array}{rrr}
-u & 0 & 0 \\
\hat{w} & u & 0 \\
0 & 0 & u
\end{array}\right)
$$

leads to

$$
P=\left(\begin{array}{ccc}
-\left(\frac{1}{1-M^{2}}+1\right) & \frac{M}{1-M^{2}} & 0 \\
\frac{M}{1-M^{2}} & \frac{-M^{2}}{1-M^{2}} & 0 \\
0 & 0 & 1
\end{array}\right)
$$

Note the resemblance between (27) and (24). When we interpret the postconditioning matrix (27) as a solution transformation matrix $\mathrm{d} \tilde{q} / \mathrm{d} q$, we get

$$
\mathrm{d} \tilde{q}=\left(\begin{array}{c}
-\frac{M^{2}-2}{M^{2}-1} \frac{1}{\rho c} \mathrm{~d} p-\frac{M}{M^{2}-1} \mathrm{~d} u \\
-\frac{M}{M^{2}-1} \frac{1}{\rho c} \mathrm{~d} p+\frac{M^{2}}{M^{2}-1} \mathrm{~d} u \\
\mathrm{~d} p-c^{2} \mathrm{~d} \rho
\end{array}\right) .
$$

Physical interpretation of the first two components of $\mathrm{d} \tilde{q}$ is not trivial. In the remainder we consider preconditioning according to (24). Concerning the relevance of doing 1-D analyses with (24) for multi-D preconditioned equations, the following can be remarked. The 1-D preconditioning matrix (24) resembles the 2-D preconditioning matrix (21). In 2-D (and also in 3-D) preconditioning is done for the Euler equations which are locally rotated in streamwise direction. So, in fact, in multi-D preconditioning is done for quasi-1-D equations. Therefore we expect that the 1-D analyses done in this paper are relevant for the multi-D preconditioned equations.

\subsection{Concerning inaccuracy}

A partial fix to the discrete accuracy problem discussed in section 4.2 , is to make the discretization second-order accurate. If one applies a second-order accurate discretization, the error still diverges for $M \downarrow 0$, but not as soon as in the case of a firstorder discretization (because of the $h^{2}$-instead of the $h$-proportionality). Of course, as long as the two limits $M \downarrow 0$ and $h \downarrow 0$ are independent (and as long as the discretization method is not exact), formally the accuracy problem continues to exist. A subsequent fix would then be to take the mesh size appropriately dependent on the Mach number. 
A really good remedy is to exploit the freedom still existing in the choice of the preconditioning matrices for removing the stiffness. By first preconditioning:

$$
\frac{\partial q}{\partial t}+P A \frac{\partial q}{\partial x}=0
$$

and next discretizing, one gets the influence of the preconditioning in the discretization error. For flow computations at uniformly low Mach numbers, the challenge is then to get rid of both the stiffness and the accuracy problem by a single preconditioning matrix $P$. It has been shown, both in practice [14] and in theory [24], that the matrices (21) and (24) accomplish this. Discretization of (29) requires the incorporation of a space discretization scheme which is modified for the preconditioning (both at the interior and the boundary cell faces). Further, in multigrid contexts the residual transfer has to be reconsidered, in order to maintain the Galerkin property and hence good multigrid convergence [12]. Since uniformly low-Mach-number flows are not our present interest, we will not apply the preconditioning in the form (29). (For computations in which the Mach number is not uniformly low, the accuracy problems occurring for $\lim _{M \downarrow 0}$ are local, and hence no reduction of global solution accuracy is expected to be found.)

\section{Implementing the preconditioning}

By implementing the preconditioning as

$$
P^{-1} \frac{\partial q}{\partial t}+A \frac{\partial q}{\partial x}=0
$$

with $P^{-1}$ the inverse of (24):

$$
P^{-1}=\left(\begin{array}{ccc}
\frac{2-M^{2}}{M^{2}} & \frac{1}{M} & 0 \\
\frac{1}{M} & 1 & 0 \\
0 & 0 & 1
\end{array}\right)
$$

the original space discretization scheme can still be applied (because the space operator is still original). Steady-state solutions will therefore be identical to those belonging to the unconditioned equations (5a) (and (15)). The conservative form corresponding to $(30 \mathrm{a})$ reads

$$
\frac{\mathrm{d} Q}{\mathrm{~d} q} P^{-1} \frac{\partial q}{\partial t}+\frac{\partial f(Q)}{\partial x}=0
$$

Taking the integral form of (31), discretizing that by a first-order upwind finitevolume method, and denoting the numerical flux function which approximates 
cell-face flux $f\left(q_{i+1 / 2}\right)$ by $F\left(q_{i}, q_{i+1}\right)$, for cell $\Omega_{i}$ the semi-discrete equation reads

$$
\int_{\Omega_{i}} \frac{\mathrm{d} Q}{\mathrm{~d} q} P^{-1} \frac{\partial q}{\partial t} \mathrm{~d} x+\left(F\left(q_{i}, q_{i+1}\right)-F\left(q_{i-1}, q_{i}\right)\right)=0 .
$$

Given the good smoothing properties of point Gauss-Seidel relaxation in the multigrid computation of high-subsonic, transonic and supersonic flows, in choosing the time discretization for (32) we want to deviate as little as possible from this trusty smoother. Therefore we apply locally implicit time-stepping in a Gauss-Seidel fashion. Hence, as fully discrete equation in cell $\Omega_{i}$, for downstream and upstream sweeps, respectively, it follows:

$$
\begin{gathered}
{\left[\frac{h}{\Delta t} \frac{\mathrm{d} Q}{\mathrm{~d} q}\left(q_{i}^{n}\right) P^{-1}\left(M_{i}^{n}\right)+\frac{\partial F\left(q_{i}^{n}, q_{i+1}^{n}\right)}{\partial q_{i}^{n}}-\frac{\partial F\left(q_{i-1}^{n+1}, q_{i}^{n}\right)}{\partial q_{i}^{n}}\right]\left(q_{i}^{n+1}-q_{i}^{n}\right)} \\
=F\left(q_{i-1}^{n+1}, q_{i}^{n}\right)-F\left(q_{i}^{n}, q_{i+1}^{n}\right), \\
{\left[\frac{h}{\Delta t} \frac{\mathrm{d} Q}{\mathrm{~d} q}\left(q_{i}^{n+1}\right) P^{-1}\left(M_{i}^{n+1}\right)+\frac{\partial F\left(q_{i}^{n+1}, q_{i+1}^{n+2}\right)}{\partial q_{i}^{n+1}}-\frac{\partial F\left(q_{i-1}^{n+1}, q_{i}^{n+1}\right)}{\partial q_{i}^{n+1}}\right]\left(q_{i}^{n+2}-q_{i}^{n+1}\right)} \\
=F\left(q_{i-1}^{n+1}, q_{i}^{n+1}\right)-F\left(q_{i}^{n+1}, q_{i+1}^{n+2}\right) .
\end{gathered}
$$

The time step $\Delta t$ (which due to the preconditioning is not identical to a physical time step) is still amenable to optimization. In the next section it will be optimized for smoothing.

\section{Optimization of the locally implicit iteration step}

For simplicity, smoothing optimization of $\Delta t$ from (33a) and (33b) is done for the non-conservative, frozen-coefficient variants of both equations, i.e. for:

$$
\begin{aligned}
\left(\frac{h}{\Delta t} P^{-1}+|A|\right)\left(q_{i}^{n+1}-q_{i}^{n}\right) & =-A^{+}\left(q_{i}^{n}-q_{i-1}^{n+1}\right)-A^{-}\left(q_{i+1}^{n}-q_{i}^{n}\right) \\
\left(\frac{h}{\Delta t} P^{-1}+|A|\right)\left(q_{i}^{n+2}-q_{i}^{n+1}\right) & =-A^{+}\left(q_{i}^{n+1}-q_{i-1}^{n+1}\right)-A^{-}\left(q_{i+1}^{n+2}-q_{i}^{n+1}\right)
\end{aligned}
$$

\subsection{Qualitative optimization}

From (34a) and (34b), with (11a) and (11b), in the same way as in section 3.1, we derive:

$$
\begin{aligned}
\mathscr{M}_{\text {downstream }} & =\left(\frac{h}{\Delta t} P^{-1}-\mathrm{e}^{-\mathrm{i} \theta} A^{+}+|A|\right)^{-1}\left(\frac{h}{\Delta t} P^{-1}-\mathrm{e}^{\mathrm{i} \theta} A^{-}\right), \\
\mathscr{M}_{\text {upstream }} & =\left(\frac{h}{\Delta t} P^{-1}+\mathrm{e}^{\mathrm{i} \theta} A^{-}+|A|\right)^{-1}\left(\frac{h}{\Delta t} P^{-1}+\mathrm{e}^{-\mathrm{i} \theta} A^{+}\right) .
\end{aligned}
$$


We proceed by considering the two highest error frequencies: $|\theta|=\pi$. For both frequencies, with

$$
\sigma \equiv \frac{h}{c \Delta t}
$$

(35a) and (35b) can be written out as:

$$
\begin{aligned}
\mathscr{M}_{\text {downstream }}= & \left(\begin{array}{ccc}
\sigma \frac{2-M^{2}}{M^{2}}+\frac{3}{2}+\frac{1}{2} M & \sigma \frac{1}{M}+\frac{1}{2}+\frac{3}{2} M & 0 \\
\sigma \frac{1}{M}+\frac{1}{2}+\frac{3}{2} M & \sigma+\frac{3}{2}+\frac{1}{2} M & 0 \\
0 & 0 & \sigma+2 M
\end{array}\right)^{-1} \\
& \times\left(\begin{array}{ccc}
\sigma \frac{2-M^{2}}{M^{2}}-\frac{1}{2}+\frac{1}{2} M & \sigma \frac{1}{M}+\frac{1}{2}-\frac{1}{2} M & 0 \\
\sigma \frac{1}{M}+\frac{1}{2}-\frac{1}{2} M & \sigma-\frac{1}{2}+\frac{1}{2} M & 0 \\
0 & 0 & \sigma
\end{array}\right) \\
\mathscr{M}_{\text {upstream }}= & \left(\begin{array}{ccc}
\sigma \frac{2-M^{2}}{M^{2}}+\frac{3}{2}-\frac{1}{2} M & \sigma \frac{1}{M}-\frac{1}{2}+\frac{3}{2} M & 0 \\
\sigma \frac{1}{M}-\frac{1}{2}+\frac{3}{2} M & \sigma+\frac{3}{2}-\frac{1}{2} M & 0 \\
0 & 0 & \sigma+M
\end{array}\right)^{-1} \\
& \left(\begin{array}{ccc}
\sigma \frac{2-M^{2}}{M^{2}}-\frac{1}{2}-\frac{1}{2} M & \sigma \frac{1}{M}-\frac{1}{2}-\frac{1}{2} M & 0 \\
\sigma \frac{1}{M}-\frac{1}{2}-\frac{1}{2} M & \sigma-\frac{1}{2}-\frac{1}{2} M & 0 \\
0 & 0 & \sigma-M
\end{array}\right)^{-1}
\end{aligned}
$$

The corresponding eigenvalues are:

$$
\begin{gathered}
\left(\lambda_{1}\right)_{\mathscr{H}_{\text {dowosstram }}}=\frac{\sigma}{\sigma+2 M}, \quad\left(\lambda_{2,3}\right)_{\mathscr{M}_{\text {downstream }}}=\frac{\sigma+\sigma^{2}-M^{2} \pm \sqrt{4 \sigma^{2}\left(1-M^{2}\right)+M^{4}}}{3 \sigma+\sigma^{2}+2 M^{2}} \\
\left(\lambda_{1}\right)_{\mathscr{M}_{\text {upstream }}}=\frac{\sigma-M}{\sigma+M}, \quad\left(\lambda_{2,3}\right)_{\mathscr{M}_{\text {upstream }}}=\frac{\sigma+\sigma^{2}-M^{2} \pm \sqrt{4 \sigma^{2}\left(1-M^{2}\right)+M^{4}}}{3 \sigma+\sigma^{2}+2 M^{2}}
\end{gathered}
$$

Note that $\left(\lambda_{2,3}\right)_{\mathscr{M}_{\text {downstream }}}=\left(\lambda_{2,3}\right)_{\mathscr{M}_{\text {upstream }}}$. We proceed by considering the eigenvalues for $\lim _{M \downarrow 0}$. With $\sigma$ a finite (positive) constant this yields

$$
\begin{aligned}
& \lim _{M \downarrow 0}\left(\lambda_{1}, \lambda_{2}, \lambda_{3}\right)_{\mathscr{M}_{\text {dounstream }}, \sigma=\text { constant }}=\left(1,1, \frac{-\sigma+\sigma^{2}}{3 \sigma+\sigma^{2}}\right), \\
& \lim _{M \downarrow 0}\left(\lambda_{1}, \lambda_{2}, \lambda_{3}\right)_{\mathscr{M}_{\text {upstream }}, \sigma=\text { constant }}=\left(1,1, \frac{-\sigma+\sigma^{2}}{3 \sigma+\sigma^{2}}\right) .
\end{aligned}
$$


For $\sigma=\nu M$ with $\nu$ a finite (positive) constant it yields

$$
\begin{aligned}
& \lim _{M \downarrow 0}\left(\lambda_{1}, \lambda_{2}, \lambda_{3}\right)_{\mathscr{M}_{\text {downstream }}, \sigma=\nu M}=\left(\frac{\nu}{\nu+2}, 1,-\frac{1}{3}\right), \\
& \lim _{M \downarrow 0}\left(\lambda_{1}, \lambda_{2}, \lambda_{3}\right)_{\mathscr{M}_{\mathrm{upstream},} \sigma=\nu M}=\left(\frac{\nu-1}{\nu+1}, 1,-\frac{1}{3}\right) .
\end{aligned}
$$

Thus the choice $\sigma=$ constant yields two maximum eigenvalues equal to one, for both the downstream and upstream sweeps. For $\sigma=\nu M$ with $\nu$ constant, this number is only one, which probably implies smaller Frobenius matrix norms (see e.g. chapter 2 from [7]) and hence better smoothing when applying two, three, four, ... Gauss-Seidel sweeps. Note that no function $\sigma=\sigma(M)$ exists which makes the moduli of all three eigenvalues smaller than one for $\lim _{M \downarrow 0}$. We proceed with $\sigma=\nu M$, in the next section the optimal value of $\nu$ is derived.

\subsection{Quantitative optimization}

In order to optimize $\nu$ from $\sigma=\nu M$, we continue to apply Fourier analysis for the highest error frequencies $|\theta|=\pi$, where, as in section 3.1, the spectral radii of the amplification matrices are considered. To avoid Mach-number dependence of $\nu$, we consider the moduli of the amplification matrices' eigenvalues integrated over the entire subsonic Mach-number range. (Avoiding Mach-number dependence by taking $\lim _{M 10}$ does not allow $\nu$-optimization; from (40a) and (40b) it appears that the corresponding spectral radii of both $\mathscr{M}_{\text {downstream }}$ and $\mathscr{M}_{\text {upstream }}$ equal one, for any $\nu$.) In figure 2 the distributions of the aforementioned eigenvalue integrals are depicted over the $\nu$-range $[0,10]$. (Note that since $\left(\lambda_{2,3}\right)_{\mathscr{M}_{\text {downstream }}}=\left(\lambda_{2,3}\right)_{\mathscr{M}_{\text {upstream }}}$, the corresponding integrals are the same.) From figure 2 it can be seen that the optimal value of $\nu$ follows from

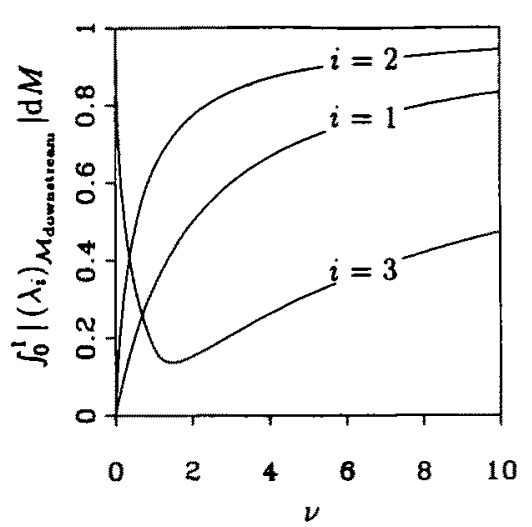

a. Downstream sweep.

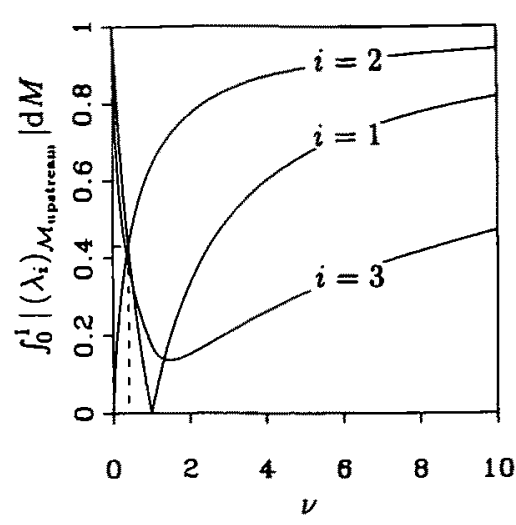

b. Upstream sweep.

Figure 2. Integrated moduli of the eigenvalues of the amplification matrices, for the highest error frequencies. 
$\int_{0}^{1}\left|\left(\lambda_{1}\right)_{\mathscr{A}_{\text {upsiream }}}\right| \mathrm{d} M=\int_{0}^{1}\left|\left(\lambda_{2}\right)_{\mathscr{M}_{\text {upsiram }}}\right| \mathrm{d} M$ (dashed line in figure $2 \mathrm{~b}$ ), i.e. (after some computer algebra) from:

$$
\begin{gathered}
\nu \frac{-4+2 \nu-2 \nu^{2}+\nu^{3}+\left(5-2 \nu^{2}\right) \ln \left(\frac{(\nu+2)^{2}}{\nu(2 \nu+1)}\right)-3 \sqrt{1-4 \nu^{2}} \ln \left(\frac{\sqrt{1-4 \nu^{2}}+1}{2 \nu}\right)}{\left(\nu^{2}+2\right)^{2}} \\
+\frac{\nu-1}{\nu+1}=0 .
\end{gathered}
$$

From (41), it follows by good approximation that $\nu=2 / 5$, and thus as (approximately) optimal $\sigma$ :

$$
\sigma=\frac{2}{5} M
$$

\section{Convergence for the preconditioned equations}

\subsection{Error smoothing}

Relation (42) implies as (approximately) optimal iteration step $\Delta t$ :

$$
\Delta t=\frac{5}{2} \frac{h}{u},
$$

i.e. $C F L=5 / 2$. We verify the smoothing behavior for this iteration step. This is done over the entire subsonic Mach-number range $(0,1)$, for the three error frequencies $\theta=\pi / 2,3 \pi / 4$ and $\pi$. In figure 3 the distributions of the corresponding spectral radii are depicted. Recalling from section 3.1 that the spectral radii of downstream and upstream point Gauss-Seidel relaxation equal one over the entire subsonic Mach-number range, from figure 3 it appears that the preconditioning does a good job.

\subsection{Error convection}

The locally implicit iteration applied to preconditioned Euler equations may be interpreted as physical time-stepping. To do so, for simplicity we consider the common $P$ according to (20) with $w=u$. Then, with $\mathrm{CFL}=u \Delta t / h$, the iteration formulae (34a) and (34b) become

$$
\begin{aligned}
(1+\mathrm{CFL})|A|\left(q_{i}^{n+1}-q_{i}^{n}\right) & =-A^{+}\left(q_{i}^{n}-q_{i-1}^{n+1}\right)-A^{-}\left(q_{i+1}^{n}-q_{i}^{n}\right), \\
(1+\mathrm{CFL})|A|\left(q_{i}^{n+2}-q_{i}^{n+1}\right) & =-A^{+}\left(q_{i}^{n+1}-q_{i-1}^{n+1}\right)-A^{-}\left(q_{i+1}^{n+2}-q_{i}^{n+1}\right) .
\end{aligned}
$$

From (44a) and (44b) it appears that for this common $P$, the locally implicit timestepping can be directly interpreted as point Gauss-Seidel relaxation with underrelaxation factor $\omega=1+$ CFL. I.e., even with CFL $=\mathcal{O}(1)$, (44a) and (44b) can 


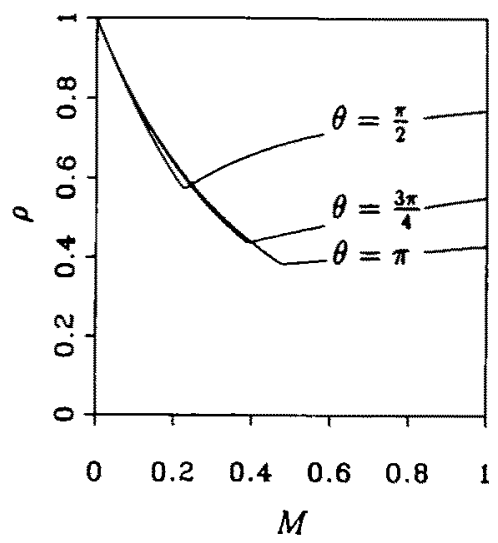

a. Downstream sweep.

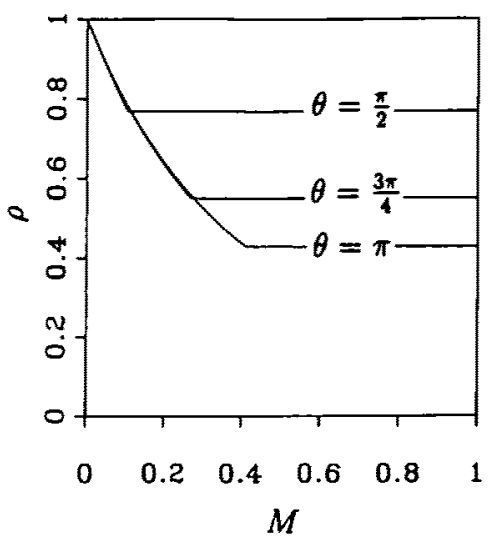

b. Upstream sweep.

Figure 3. Spectral radii of the amplification matrices, for the downstream and upstream (approximately optimal) point Gauss-Seidel "time"-stepping, and three error frequencies.

still be interpreted as locally implicit physical time-stepping at an infinitely large time step.

\section{Conclusions}

- Poor convergence of multigrid accelerated point Gauss-Seidel relaxation at low Mach numbers is explained by the relaxation's poor smoothing at low Mach numbers and by the likewise poor entropy-error expulsion across domain boundaries.

- Poor solution accuracy known to occur at low Mach numbers can be explained by means of the modified equation for the 1-D Euler equations, discretized by a first-order accurate flux-difference splitting scheme. For flows with uniformly low Mach numbers, a fix to this inaccuracy is a necessity. For flows of which the global solution error is not affected by the occurrence of low-subsonic flow regions such a fix may not be necessary.

- For the latter flows, implementation of preconditioning in a locally implicit time-stepping method with the inverse of the preconditioning matrix working on the time operator, may be practical. It allows the application of an offthe-shelf space discretization method.

- Local-mode analysis shows that optimal high-frequency damping for locally implicit "time"-stepping in a Gauss-Seidel way, is obtained for CFL $\approx 5 / 2$. (When preconditioning with the 1-D matrix $P=u|A|^{-1}$, the locally implicit "time"-stepping boils down to point Gauss-Seidel relaxation with underrelaxation factor $1+$ CFL.)

- Given the direct availability of the 2-D and 3-D extensions of the 1-D preconditioning matrix analyzed, the present improved solution method is directly extendible to multi-D. 


\section{References}

[1] L. Bers, Mathematical Aspects of Subsonic and Transonic Gas Dynamics (Wiley, London, 1958).

[2] Y.-H. Choi and C.L. Merkle, The application of preconditioning in viscous flows, J. Comp. Phys. 105 (1993) 207-223.

[3] R. Courant and K.O. Friedrichs, Supersonic Flows and Shock Waves (Springer, New York, 1976).

[4] M. Van Dyke, Perturbation Methods in Fluid Mechanics (The Parabolic Press, Stanford, 1975).

[5] C. Ferrari and F.G. Tricomi, Transonic Aerodynamics (Academic Press, New York, 1968).

[6] A. Godfrey, R.W. Walters and B. van Leer, Preconditioning for the Navier-Stokes equations with finite-rate chemistry, AIAA-93-0535 (1993).

[7] G.H. Golub and C.F. Van Loan, Matrix Computations (The John Hopkins University Press, Baltimore, 1989).

[8] K.G. Guderley, Theorie Schallnaher Strömungen (Springer, Berlin, 1957).

[9] J. Guerra and B. Gustafsson, A numerical method for incompressible and compressible flow problems with smooth solutions, J. Comp. Phys. 63 (1986) 377-397.

[10] B. Gustafsson, Unsymmetric hyperbolic systems and the Euler equations at low Mach numbers, J. Sci. Comp. 2 (1987) 123-136.

[11] B. Gustafsson and P. Lötstedt, Analysis of the multigrid method applied to first order systems, in: Proc. 4th Copper Mountain Conf. on Multigrid Methods, eds. J. Mandel et al. (SIAM, Philadelphia, PA, 1989) pp. 181-233.

[12] P.W. Hemker and S.P. Spekreijse, Multiple grid and Osher's scheme for the efficient solution of the steady Euler equations, Appl. Numer. Math. 2 (1986) 475-493.

[13] W.-T. Lee, Local preconditioning of the Euler equations, Ph.D.-thesis, The University of Michigan (1992).

[14] B. van Leer, W.-T. Lee and P.L. Roe, Characteristic time-stepping or local preconditioning of the Euler equations, AIAA-91-1552 (1991).

[15] B. van Leer, E. Turkel, C.-H. Tai and L. Mesaros, Local preconditioning in a stagnation point, in preparation.

[16] A. Majda, Compressible Fluid Flow and Systems of Conservation Laws in Several Space Variables (Springer, Berlin, 1984).

[17] R. von Mises, Mathematical Theory of Compressible Fluid Flow (Academic Press, New York, 1958).

[18] C.S. Morawetz, On the non-existence of continuous transonic flows past airfoils I, Comm. Pure Appl. Math. 9 (1956) 45-68; II, ibid. 10 (1957) 107-131; III, ibid. 11 (1958) 129-144.

[19] S. Osher and F. Solomon, Upwind difference schemes for hyperbolic systems of conservation laws, Math. Comp. 38 (1982) 339-374.

[20] K. Oswatitsch, Spezialgebiete der Gasdynamik; Schallnahe, Hyperschall, Tragflachen, Wellenausbreitung (Springer, Wien, 1977).

[21] P.L. Roe, Approximate Riemann solvers, parameter vectors, and difference schemes, J. Comp. Phys. 43 (1981) 357-372.

[22] E. Turkel, Preconditioned methods for solving the incompressible and low speed compressible equations, J. Comp. Phys. 72 (1987) 277-298.

[23] E. Turkel, Review of preconditioning methods for fluid dynamics, Appl. Numer. Math. 12 (1993) 257-284.

[24] E. Turkel, A. Fiterman and B. van Leer, Preconditioning and the limit of the compressible to the incompressible flow equations for finite difference schemes, in: Computing the Future: Advances and Prospects for Computational Aerodynamics, eds. M.M. Hafez and D.A. Caughey (Wiley, Chichester, 1994) pp. 215-234.

[25] G. Volpe, Performance of compressible codes at low Mach numbers, AIAA J. 31 (1993) 49-56.

[26] P. Wesseling, An Introduction to Multigrid Methods (Wiley, Chichester, 1992). 